\title{
P-wave Analysis in Atrial Fibrillation Detection Using a Neural Network Clustering Algorithm
}

\author{
Reza Firoozabadi, Richard E Gregg, Saeed Babaeizadeh
}

\author{
Advanced Algorithm Research Center, Philips Healthcare, Andover, MA, USA
}

\begin{abstract}
Absence of $P$-waves in ECG records with irregular interbeat intervals $(R-R)$ is a sign of Atrial Fibrillation $(A F)$. Detection of $P$-waves in ECG beats or even average beats could be challenging if the artifact resembles a $P$ wave, or an actual P-wave is buried in the artifact. We developed a neural network algorithm to generate the ECG beat clusters in segments of the record. Beats with matching QRS complexes were clustered using SelfOrganizing Map (SOM) technique and then crosscorrelated to combine and generate the dominant clusters. This process helps to eliminate the abnormal or artifactcorrupted beats. Fiducial points of the dominant average beat were measured by morphological techniques. If the Pwave was detected in the average beat, a smaller search window was defined for individual beats to exclude the potentially false $P$-waves. A set of $P$-wave features determined the presence of $P$-wave throughout an ECG segment. Our algorithm was tested on several datasets with annotated intervals for some cardiac rhythms. A decision tree ensemble of bagged trees classifier was developed and applied to the $P$-wave and interbeat interval features, resulting in AF/non-AF classification with average $F_{1}$ score of $96.0 \%$ in training subset and $95.6 \%$ in test subset of all records.
\end{abstract}

\section{Introduction}

Atrial Fibrillation (AF) is the most common sustained cardiac arrhythmia associated with high rate of morbidity and mortality. AF is seen in about $1-2 \%$ of population and its prevalence is increasing rapidly [1,2]. Early detection and treatment of AF can prevent its subsequent complications such as stroke, heart failure, and sudden death [3].

Silent (asymptomatic) AF is associated with the same risk of cardiac diseases as symptomatic AF [4,5]. Unless identified incidentally in the patient's short ECG record, asymptomatic AF requires prolonged ECG monitoring with Holters or wearables which could be expensive and uncomfortable.

Several automated AF detection algorithms have been suggested in the past, however, automatic AF detection is still a challenging task, mostly due to its episodic nature.

Real-time data analysis techniques have dramatically evolved in recent years because of the advent of powerful processors in devices such as smartphones. Numerous machine learning and deep learning algorithms have been developed to analyze the increasing amount of data including real-time clinical measurements and physiological signals. Traditional machine learning algorithms analyze the data using a set of features designed by experts. Artificial neural networks utilizing deep learning methods can analyse a large set of data without the prerequisite to define a feature.

The 2016 European guidelines for the management of atrial fibrillation [6] recommends the diagnosis of AF in ECG by detecting its typical pattern which is completely irregular interbeat intervals between the beats with no distinguishable $\mathrm{P}$-waves in an episode lasting at least 30 seconds.

In AF detection studies, ventricular response has been analyzed using the interbeat interval irregularity features including the interbeat statistical measures [7-8] and entropy [9], while the atrial activity associated with the absence of P-waves is extracted from morphology [10] as well as wavelet analysis techniques [11].

In this study, we first utilized an unsupervised neural network algorithm to cluster the ECG beats of the same type and eliminate the abnormal or artifact-corrupted beats in the 30-sec segments of the ECG intervals annotated with the same rhythm. Secondly, we sought the potential Pwaves in the cluster average beat and each beat in the dominant rhythm cluster, which in turn defined a set of atrial activity features applied to the classifier along with the ventricular activity (interbeat intervals) features. A decision tree ensemble classifier of bagged trees was used to detect the AF rhythm in the ECG segments and measure the classification performance.

The rest of this paper is organized as follows. In Section 2 , we describe the algorithm and the database, and discuss the details of the beat clustering algorithm, P-wave detection, feature extraction, and classification method. Section 3 presents the results. Section 4 provides the discussion and conclusions. 


\section{Method and Material}

\subsection{Algorithm}

In this subsection, we first explain our unsupervised neural network algorithm, generating clusters of ECG beats, and find dominant combined clusters. We will then present our morphological P-wave detection technique which seeks the $\mathrm{P}$-wave fiducial points in the average beat and every beat in the dominant cluster. A set of P-wave features are then defined along with the $\mathrm{NN}$ interval features. Last step is AF classification of the segments using a decision tree ensemble of bagged trees.

\subsubsection{Beat Clustering}

We split the datasets of ECG records with expertannotated rhythm intervals into 30 -sec non-overlapping segments to generate a database of known cardiac rhythms with fixed length. The intervals shorter than 30 seconds were discarded. Using our QRS peak detection algorithm, we identified the QRS peaks in the 30-sec ECG segments to form a set of beats aligned at their QRS peaks.

We used a Self-Organizing Map (SOM) [12] neural network algorithm to cluster the aligned ECG beats with matching QRS complexes in Matlab. A $3 \times 3$ network of clusters was generated with each neuron containing a set of matched beats. Figure 1 shows an example of generating QRS-matched beat clusters corresponding to the neurons in the SOM network. Figure 2(a) shows the topology of SOM network of neurons (sample hits) in the same example with the number of matched beats on each neuron. Figure 2(b) shows the SOM neighbor distance map of the example where the direct neighbor connections are shown by lines between neurons. The color of the patches containing the connecting lines is proportional to the weight vector distance between adjacent neurons. Darker colors represent larger distances. Groups of similar neurons (beat clusters) are bonded by light patches, separated by darker patches from other groups of neurons.

Dominant cluster (with highest number of beats) was merged with other clusters if high correlation was measured between their average beats. In Figures 1 and 2(a), cluster 5 had the highest number of beats and high correlation with clusters $3,6,7,8$, and 9 . The method was applied to remaining clusters to find the secondary dominant cluster (cluster 1 in this example). The merged dominant cluster with non-ectopic rhythm was selected for further analysis. Using this approach, artifact-corrupted and abnormal beats were automatically excluded from the dominant cluster. Figure 3 shows (a) the dominant and (b) the subordinate average beats.

\subsubsection{P-wave Detection}

Fiducial points of the dominant average beat were measured by morphological techniques after smoothing slightly by a moving average filter. R-wave peak was already found by our QRS detector. Q peak (if available) and $\mathrm{Q}_{\mathrm{o}}(\mathrm{QRS}$ onset) were determined by maximum vertical distance method. $\mathrm{P}_{\mathrm{p}}$ (P-wave peak), if available, was then identified as the positive or negative prominent peak in a search window, then $\mathrm{P}_{\mathrm{o}}$ and $\mathrm{P}_{\mathrm{e}}$ ( $\mathrm{P}$-wave onset and end, respectively) were found by maximum vertical distance method with $\mathrm{P}_{\mathrm{p}}$ at one end of the search line. Figure 4 shows an example of our P-wave detection method.
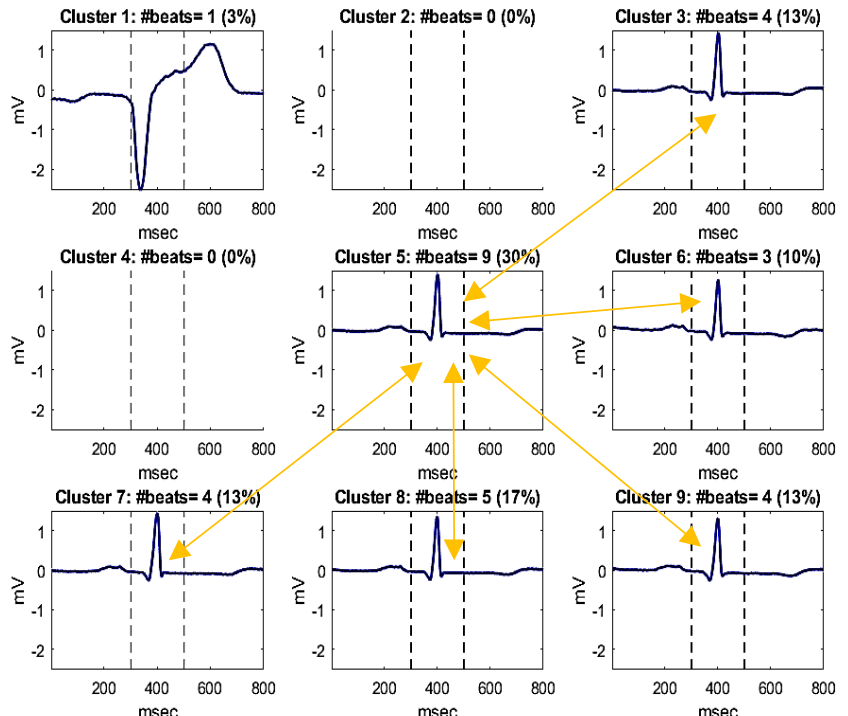

Figure 1. An example of clustering the beats by QRS matching and merging the highly-correlated cluster.

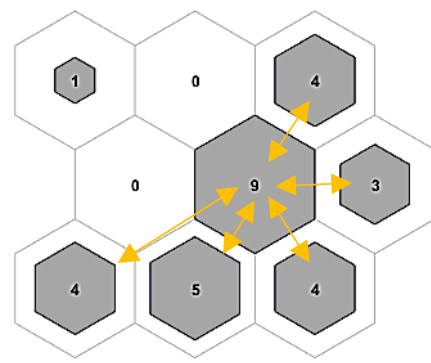

(a)

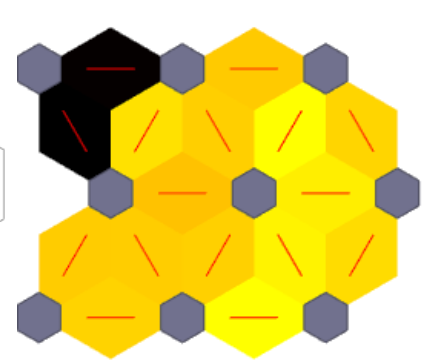

(b)
Figure 2. (a) Network topology, and (b) SOM neighbor distance map for the clusters in the example of Figure 1.

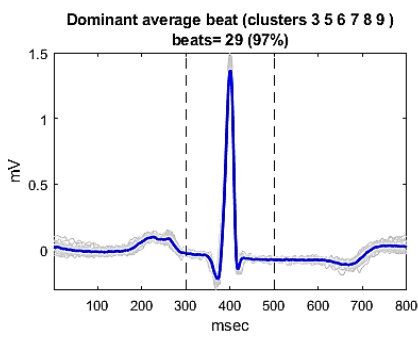

(a)

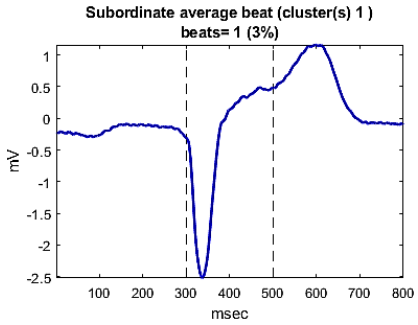

(b)
Figure 3. (a) Dominant average beat after merging similar clusters, (b) subordinate average beat from a single PVC. 


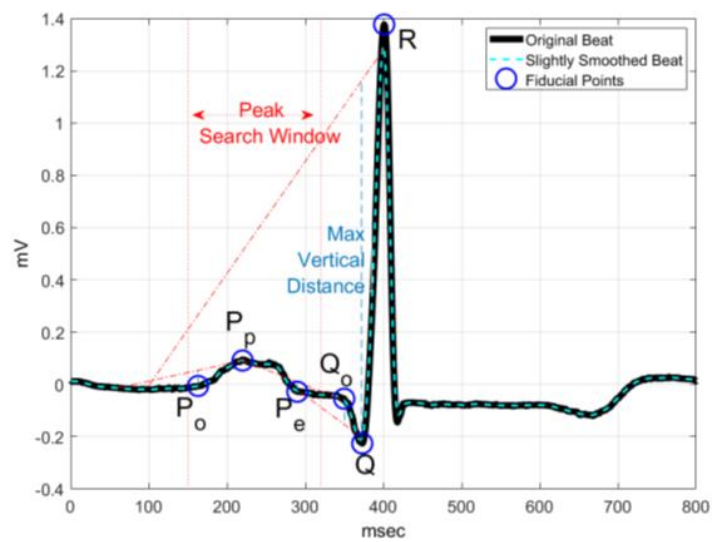

Figure 4. An example of our P-wave detection method.

To find a peak in each beat in the dominant cluster, the same technique is applied, however, for an existing P-wave in the dominant average beat, the algorithm will apply a smaller peak search window which helps to exclude the potentially false $\mathrm{P}$-waves.

\subsubsection{Feature Extraction}

The atrial activity features are associated with presence or absence of consistent P-waves in 30-sec segments of ECG recording while the ventricular response features define irregularity of the beats.

\section{Atrial activity features $(n=12)$ :}

- Mean and standard deviation of the following measures within the segment: PR interval, P-wave duration, P-wave onset-peak duration, P-wave amplitude (peak-onset)

- Number of P-waves detected in segment

- Presence/absence of potential P-wave in average beat

- Mean and standard deviation of the correlation of Pwaves in average beat with each beat in the segment.

Ventricular response features $(n=12)$ :

- Median, standard deviation, sample entropy, and Shannon entropy of each of the following measures: $\mathrm{NN}$ intervals, absolute value of the differences of the $\mathrm{NN}$ intervals, absolute value of the second differences of the NN intervals.

\subsubsection{Classifier}

Classification was performed between $\mathrm{AF}$ records and non-AF records including normal sinus rhythm (NSR) and some P-wave-bearing arrhythmias such as sinus bradycardia (SB), LBBB, RBBB, and ectopic rhythms (bigeminy and trigeminy) with normal beats used in the feature extraction.

We designed a decision tree ensemble classifier with bootstrap aggregation (bagging) with 50 decision trees. Positive event was the AF rhythm detection in segments. Each rhythm class in each database was randomly split into
$80 \%$ training data and $20 \%$ test data. We used 5-fold crossvalidation for performance evaluation of the training data. Feature importance was evaluated in the training data.

\subsection{Database}

Our algorithm was tested on several datasets with annotated intervals for some cardiac rhythms. Each 30-sec segment in these intervals was resampled to $1000 \mathrm{~Hz}$. Only the first lead was used in a multi-lead record.

The first dataset was PhysioNet MIT-BIH Arrhythmia database [13,14] including long recordings from 48 patients, originally sampled at $360 \mathrm{~Hz}$. The second dataset was the European ST-T Database $[15,16]$, collected from 79 ambulatory patients with a sample rate of $250 \mathrm{~Hz}$. The third dataset was the AHA database with 154 recordings recorded at $250 \mathrm{~Hz}$ [17]. The fourth dataset was the PhysioNet/CinC Challenge 2017 training dataset [18] collected from AliveCor ECG recording devices, consisting of 8,528 ECG recordings from 9 to 60 seconds in length at the original sample rate of $300 \mathrm{~Hz}$. We excluded the 'others' and 'noisy' classes of the challenge dataset as they did not fit in our AF/non-AF classification. Table 1 shows the number of segments in each rhythm class for all datasets.

Table 1. Number of the 30 -sec segments for each annotated rhythm in the datasets

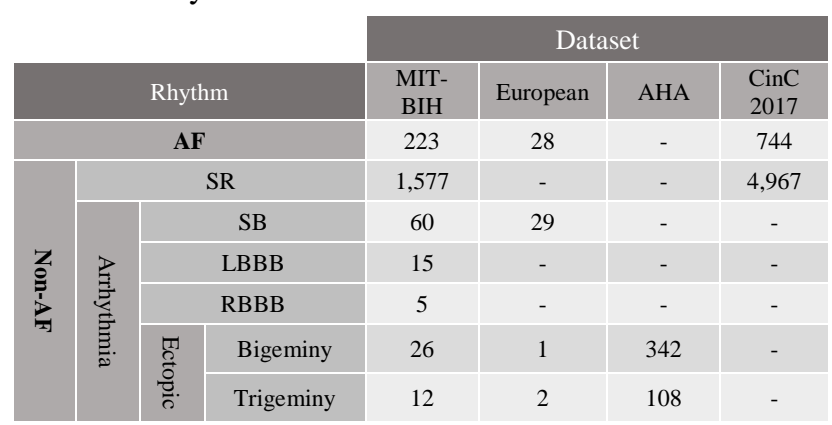

\section{Results}

Different combinations of rhythm classes and datasets were tested using our AF/non-AF classifier. Training performance was evaluated by a 5-fold cross-validation on $80 \%$ of all data, while test performance was measured on the remaining 20\%. Table 2 shows the training and test classifier performance for the combination of MIT-BIH, European, and AHA datasets, versus the CinC challenge 2017 dataset, as well as all datasets combined.

Assessment of the important features shows that in the CinC challenge 2017 dataset, ventricular response features were more important, leading with the Shannon entropy of the differences of NN intervals. Atrial activity features were more important in the group of the other three datasets with P-wave amplitude as the most important feature. Shannon entropy of differences of the NN intervals 
was the most important feature in classification of all datasets combined.

Table 2. AF/non-AF classifier performance.

\begin{tabular}{|c|c|c|c|c|c|c|c|c|c|}
\hline \multicolumn{2}{|c|}{ Datasets } & $\begin{array}{c}\text { SE } \\
(\%)\end{array}$ & $\begin{array}{c}\text { SP } \\
(\%)\end{array}$ & $\begin{array}{c}\text { PPV } \\
(\%)\end{array}$ & $\begin{array}{c}\text { NPV } \\
(\%)\end{array}$ & $\begin{array}{c}\text { F1 } \\
\text { NonAF } \\
(\%)\end{array}$ & $\begin{array}{c}\text { F1 } \\
\text { AF } \\
(\%)\end{array}$ & $\begin{array}{c}\text { F1 } \\
\text { Total } \\
(\%)\end{array}$ & $\begin{array}{c}\text { Acc } \\
(\%)\end{array}$ \\
\hline $\begin{array}{c}\text { MIT-BIH+ } \\
\text { European+ }\end{array}$ & Train. & 98.0 & 99.9 & 99.0 & 99.8 & 99.8 & 98.5 & 99.2 & 99.7 \\
\hline $\begin{array}{c}\text { AHA } \\
\text { CinC }\end{array}$ & Test & 93.9 & 100.0 & 100.0 & 99.3 & 99.7 & 96.8 & 98.3 & 99.4 \\
\hline $\begin{array}{c}\text { Challenge } \\
2017\end{array}$ & Train. & 88.4 & 99.0 & 93.1 & 98.3 & 98.7 & 90.7 & 94.7 & 97.6 \\
\hline Tll datasets & Train. & 91.4 & 99.3 & 94.7 & 98.8 & 99.0 & 93.0 & 96.0 & 98.3 \\
\hline & Test & 90.4 & 99.2 & 94.2 & 98.7 & 99.0 & 92.2 & 95.6 & 98.2 \\
\hline
\end{tabular}

\section{Discussion and Conclusions}

We performed a successful classification of AF and non-AF rhythms in four datasets containing AF, NSR, and several other arrhythmia types. Using a beat clustering neural network algorithm helped us to exclude the abnormal, dissimilar, or artifact-corrupted beats. Our Pwave detection method found the fiducial points in all beats and the average beat of the dominant cluster, and atrial activity features were defined based on the location and amplitude of these points.

We observed that the classification performance was lower for CinC challenge 2017 dataset versus the combination of other three datasets. The important features in the challenge dataset were also different form the other datasets: Ventricular response features versus atrial activity features, respectively. These differences could be due to the lower signal quality in the challenge records where the QRS-peak detection driving NN-interval was more robust than $\mathrm{P}$-wave detection.

In this work, we aimed to study the relative contribution of features defined by $\mathrm{P}$-waves and $\mathrm{NN}$-intervals. Adding more features would increase the AF/non-AF classification performance and make the classification of other arrhythmias possible. Also, signal quality analysis was out of the scope of this study.

The rhythm classes in the datasets were unbalanced in number of records and biased towards the NSR rhythms. Addition of more records with $\mathrm{AF}$ and other Non-AF rhythm classes would result in a more robust classification.

\section{References}

[1] Chugh SS, Havmoeller R, Narayanan K, et al. Worldwide epidemiology of atrial fibrillation: a Global Burden of Disease 2010 Study. Circulation 2014;129:837-847.

[2] Colilla S, Crow A, Petkun W, Singer DE, Simon T, Liu $X$. Estimates of current and future incidence and prevalence of atrial fibrillation in the U.S. adult population. Am J Cardiol 2013;112:1142-7.

[3] Wolf P A, Abbott R D, Kannel W B. Atrial fibrillation as an independent risk factor for stroke: the Framingham Study. Stroke. 1991 Aug;22 (8):983-8.

[4] Barbarossa A, Guerra F, Capucci A. Silent atrial fibrillation: A critical review. Journal of Atrial Fibrillation. 2014;7(3):1138.

[5] Healey Jeff S, Connolly Stuart J, Gold Michael R, et al. Subclinical atrial fibrillation and the risk of stroke. N. Engl. J. Med. 2012 Jan 12;366 (2):120-9.

[6] Kirchhof P, Benussi S, Kotecha D, et al. 2016 ESC Guidelines for the management of atrial fibrillation developed in collaboration with EACTS. European Heart Journal. 2016;37(38):2893-962.

[7] Moody G. A new method for detecting atrial fibrillation using RR intervals. In Computers in Cardiology. IEEE, 1983; 227-30.

[8] Costa MD, Davis RB, Goldberger AL. Heart rate fragmentation: A new approach to the analysis of cardiac interbeat interval dynamics. Frontiers in physiology 2017;8.

[9] Shannon C. A mathematical theory of communication. The Bell System Technical Journal 1948;27(3):379423.

[10] Ladavich S, Ghoraani B. Rate-independent detection of atrial fibrillation by statistical modeling of atrial activity. Biomedical Signal Processing and Control. 2015 Apr 30;18:274-81.

[11] García M, Ródenas J, Alcaraz R, Rieta JJ. Application of the relative wavelet energy to heart rate independent detection of atrial fibrillation. computer methods and programs in biomedicine 2016;131:157-68.

[12] Kohonen, T. Self-Organizing Maps. Series in Information Sciences, Vol. 30. Springer, Heidelberg. Second ed. 1997.

[13] MIT-BIH Arrhythmia Database [Online]. Available: https://physionet.org/physiobank/database/mitdb/

[14] Moody GB, Mark RG. The impact of the MIT-BIH Arrhythmia Database. IEEE Eng in Med and Biol 20(3):45-50 (May-June 2001). (PMID: 11446209)

[15] European ST-T Database [Online]. Available: https://physionet.org/physiobank/database/edb/.

[16] Taddei A, Distante G, Emdin M, et al. The European ST-T Database: standard for evaluating systems for the analysis of ST-T changes in ambulatory electrocardiography. European Heart Journal 13: 116472 (1992).

[17] AHA Database [Online]. Available: https://physionet.org/physiobank/database/ahadb/

[18] "AF Classification from a short single lead ECG recording: the PhysioNet/Computing in Cardiology Challenge 2017," 2017. [Online]. Available: https://physionet.org/challenge/2017/.

Address for correspondence:

Reza Firoozabadi

Philips Healthcare

3000 Minuteman Rd, Andover, MA 01810

reza.firoozabadi@philips.com 